\title{
FAR-IR GALACTIC EMISSION MAP AND COSMIC OPTICAL BACKGROUND
}

\author{
Y. MATSUOKA \\ Graduate School of Science, Nagoya University, Nagoya 464-8602, Japan \\ E-mail: matsuoka@a.phys.nagoya-u.ac.jp \\ (Received March 14, 2012; Accepted July 27, 2012)
}

\begin{abstract}
We present new constraints on the cosmic optical background (COB) obtained from an analysis of the Pioneer 10/11 Imaging Photopolarimeter (IPP) data. After careful examination of the data quality, the usable measurements free from the zodiacal light are integrated into sky maps at the blue $(\sim 0.44 \mu \mathrm{m})$ and red $(\sim 0.64 \mu \mathrm{m})$ bands. Accurate starlight subtraction was achieved by referring to all-sky star catalogs and a Galactic stellar population synthesis model down to 32.0 mag. We find that the residual light is separated into two components: one component shows a clear correlation with the thermal $100 \mu \mathrm{m}$ brightness, whilst the other shows a constant level in the lowest $100 \mu \mathrm{m}$ brightness region. The presence of the second component is significant after all the uncertainties and possible residual light in the Galaxy are taken into account, thus it most likely has an extragalactic origin (i.e., the COB). The derived COB brightness is $(1.8 \pm 0.9) \times 10^{-9}$ and $(1.2 \pm 0.9) \times 10^{-9} \mathrm{erg} \mathrm{s}^{-1} \mathrm{~cm}^{-2} \mathrm{sr}^{-1} \AA^{-1}$ in the blue and red spectral regions, respectively, or $7.9 \pm 4.0$ and $7.7 \pm 5.8 \mathrm{nW} \mathrm{m}^{-2} \mathrm{sr}^{-1}$. Based on a comparison with the integrated brightness of galaxies, we conclude that the bulk of the COB is comprised of normal galaxies which have already been resolved by the current deepest observations. There seems to be little room for contributions from other populations including "first stars" at these wavelengths. On the other hand, the first component of the IPP residual light represents the diffuse Galactic light (DGL) - scattered starlight by the interstellar dust. We derive the mean DGL-to-100 $\mu \mathrm{m}$ brightness ratios of $2.1 \times 10^{-3}$ and $4.6 \times 10^{-3}$ at the two bands, which are roughly consistent with previous observations toward denser dust regions. Extended red emission in the diffuse interstellar medium is also confirmed.
\end{abstract}

Key words: cosmology: observations; dark matter; diffuse radiation; dust, extinction; galaxies: evolution; infrared: ISM

\section{INTRODUCTION}

The cosmic optical background (COB) is the optical component of the extragalactic background light referring to the integrated radiation from all light sources outside the Galaxy. The dominant contribution to the COB comes from stellar nucleosynthesis in galaxies at redshifts $z<10$, while other mechanisms such as mass accretion onto supermassive black holes, gravitational collapse of stars, and particle decay can contribute to the COB brightness. As a fossil record of light production activity in the Universe, the COB conveys information on the cosmic star formation history, including the birth and death of Population III stars. In this regard, $\mathrm{COB}$ measurements provide complementary information to that of studies of individual galaxies (Matsuoka et al., 2010, 2011b), active galactic nuclei (Matsuoka et al., 2007, 2008, 2012), and other populations. However, robust detection of the COB has long been hampered by the extremely bright foreground emissions. While the expected brightness of the COB is around $1 \mathrm{bgu} \equiv$ $1 \times 10^{-9} \mathrm{erg} \mathrm{s}^{-1} \mathrm{~cm}^{-2} \mathrm{sr}^{-1} \AA^{-1}$, the terrestrial airglow and the zodiacal light (ZL) are several orders of magnitude brighter than this level at optical wavelengths (Leinert et al., 1998). In addition, the diffuse Galactic 
light (DGL), which refers to the starlight scattered by the interstellar dust, contributes another, much fainter component to the night sky. In order to overcome the long-standing problem of foreground removal, we have made use of imaging data obtained from the Imaging Photopolarimeters on the Pioneer 10 and 11 spacecraft (Matsuoka et al., 2011a). The methodology and results are presented here.

\section{OBSERVATIONS AND REDUCTIONS}

A detailed description of the observations and of the primary data processing can be found in, e.g., Weinberg et al. (1974) and Gordon et al. (1998). Using the Pioneer 10 IPP data collected at various heliocentric distances $R$, Hanner et al. (1974) found that the ZL brightness is below the detectable level of the instrument when the spacecraft was at beyond $R=3.26$ AU. Hence the data obtained at the larger distances are most suitable for an analysis of diffuse radiation originating outside of the ZL clouds.

We thoroughly cleaned the data by removing those with negative flux levels, possible contamination of the scattered sunlight, and abnormal values of fluxes and/or colors. All of the good-quality data were then integrated into a single sky map at each of the IPP blue $\left(B_{\mathrm{IPP}}\right)$ and red $\left(R_{\mathrm{IPP}}\right)$ bands, using a similar algorithm to the 1st iteration of the maximum correlation method described in Aumann et al. (1990). The final maps have an angular resolution of $\sim 0.7^{\circ}$. The possible systematic uncertainties were estimated by dividing the data into various subgroups with several criteria such as spacecrafts and heliocentric distances and comparing the sky maps created from each of them. The full description of the data reduction process is given in Matsuoka et al. (2011a).

\section{GALACTIC LIGHT}

Outside of the detectable ZL clouds, the dominant brightness component incident on the IPPs is that of the Galactic starlight. Since the contribution due to the brightest stars has already been subtracted when the archival data were created, we subtracted the contribution of fainter stars. The integrated brightness of relatively bright $(V<11 \mathrm{mag})$ and faint $(V>11 \mathrm{mag})$ stars were calculated from the Tycho-2 Catalog (Høg et al., 2000) and the Hubble Space Telescope (HST) Guide Star Catalog II (GSC-II) version 2.3 (Lasker et al., 2008), respectively. Furthermore, the contribution of stars even fainter than the GSC-II detection limits was estimated, down to $32.0 \mathrm{mag}$, using a star count model provided by the stellar population synthesis code TRILEGAL (Girardi et al., 2005). Transformations of stellar magnitudes in the different passband systems were derived using the Bruzual-Persson-GunnStryker (BPGS) stellar spectral atlas. We integrated the starlight-subtracted IPP brightness into sky maps with the same algorithm as described above, which gave us the diffuse emission maps consisting of diffuse Galactic and extragalactic emission components.

The DGL is attributed to dust and gas particles in the interstellar medium (ISM). Dominant contribution to the optical DGL comes from scattering of the interstellar radiation field (ISRF) by the dust. We estimated the DGL contribution to IPP diffuse emission brightness by separating out the component which correlates with the diffuse Galactic far-IR emission. The good correlation between the DGL and far-IR emission brightness is a natural result from the fact that both emissions are caused by the interstellar dust exposed to the ISRF, and is actually observed in several regions (compiled in Bernstein et al., 2002). With the DGLto-100 $\mu \mathrm{m}$ brightness ratio $a_{\mathrm{d}}$, the IPP diffuse emission brightness $S^{\text {diffuse }}$ can be written as:

$$
\begin{aligned}
S^{\text {diffuse }} & =S^{\mathrm{DGL}}+S^{\mathrm{COB}} \\
& =a_{\mathrm{d}}\left(S_{100 \mu \mathrm{m}}^{\text {diffuse }}-S_{100 \mu \mathrm{m}}^{\mathrm{CIB}}\right)+S^{\mathrm{COB}}
\end{aligned}
$$

where $S^{\mathrm{DGL}}$ and $S^{\mathrm{COB}}$ are the DGL and COB brightness at the IPP bands, and $S_{100 \mu \mathrm{m}}^{\text {diffuse }}$ is diffuse $100 \mu \mathrm{m}$ brightness observed outside the ZL clouds. The cosmic infrared background (CIB) component $S_{100 \mu \mathrm{m}}^{\mathrm{CIB}}$ is subtracted from $S_{100 \mu \mathrm{m}}^{\text {diffuse }}$ in the above equation, leaving only the Galactic component.

\section{RESULTS AND DISCUSSION}

We show the measured IPP diffuse emission brightness $S^{\text {diffuse }}$ versus the diffuse $100 \mu \mathrm{m}$ brightness $S_{100 \mu \mathrm{m}}^{\text {diffuse }}$ outside the ZL clouds in Figure 1, at $B_{\text {IPP }}$ band as an example. Our analysis focuses on the lowest brightness region with $S_{100 \mu \mathrm{m}}^{\text {diffuse }}<3.0 \mathrm{MJy} \mathrm{sr}^{-1}$ and with the IPP map coverage at the Galactic latitudes $|b|>35^{\circ}$, which corresponds to about a quarter of the whole sky. The diffuse $100 \mu \mathrm{m}$ brightness is taken from Schlegel et al. (1998). We clearly detect the linear correlations between $S^{\text {diffuse }}$ and $S_{100 \mu \mathrm{m}}^{\text {diffuse }}$ at large $S_{100 \mu \mathrm{m}}^{\text {diffuse }}$, and more importantly, the flattening of these relations at 


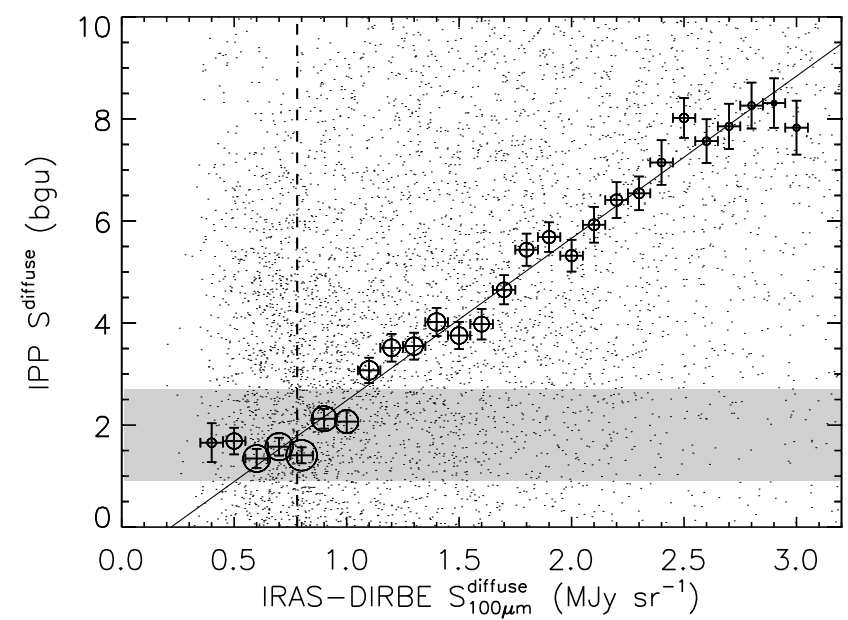

Fig. 1. Observed IPP diffuse emission brightness $S^{\text {diffuse }}$ at $B_{\text {IPP }}$ versus the diffuse $100 \mu \mathrm{m}$ brightness $S_{100 \mu m}^{\text {diffuse }}($ dots). The open circles and error bars represent mean values of $S^{\text {diffuse }}$ and their errors in the $S_{100 \mu m}^{\text {diffuse }}$ bins. The sizes of the circles are proportional to numbers of data points in the bins. The solid line shows the regression line at $S_{100 \mu m}^{\text {diffuse }}>$ 1.0 $\mathrm{MJr} \mathrm{sr}^{-1}$, while the dashed line shows the CIB brightness $S_{100 \mu m}^{\mathrm{CIB}}$ reported by Lagache et al. (2000). The shaded area shows the $1 \sigma$ confidence interval of our final COB estimate.

$S_{100 \mu \mathrm{m}}^{\text {diffuse }}<0.8 \mathrm{MJy} \mathrm{sr}^{-1}$ in both the IPP bands. The inflection points are in very good agreement with the CIB brightness reported by Lagache et al. (2000), which had been predicted in a Monte-Carlo simulation (Matsuoka et al., 2011a). Our estimates of the COB brightness are derived as the $S^{\text {diffuse }}$ values on the observed $S^{\text {diffuse }}-S_{100 \mu \mathrm{m}}^{\text {diffuse }}$ linear correlations made by the DGL, at the point where $100 \mu \mathrm{m}$ brightness equals to the CIB (i.e., $S_{100 \mu \mathrm{m}}^{\text {diffuse }}=S_{100 \mu \mathrm{m}}$ in Equation 1). Taking all the uncertainties in the measurement and data reduction processes into account, the obtained results are $S^{\mathrm{COB}}$ $=1.8 \pm 0.9$ and $1.2 \pm 0.9 \mathrm{bgu}$, or $7.9 \pm 4.0$ and 7.7 $\pm 5.8 \mathrm{nW} \mathrm{m}^{-2} \mathrm{sr}^{-1}$, in the $B_{\mathrm{IPP}}$ and $R_{\mathrm{IPP}}$ bands, respectively.

We have compiled the current measurements of the cosmic background and the integrated brightness of galaxies at ultraviolet, optical, and near-IR wavelengths in Figure 2. By contrast to the results from Bernstein (2007), our new results of the COB brightness are mildly larger than and consistent within $1 \sigma$ uncertainty level with the integrated brightness of galaxies measured in the Hubble deep field (HDF; Madau \& Pozzetti, 2000). They are on the smooth extension

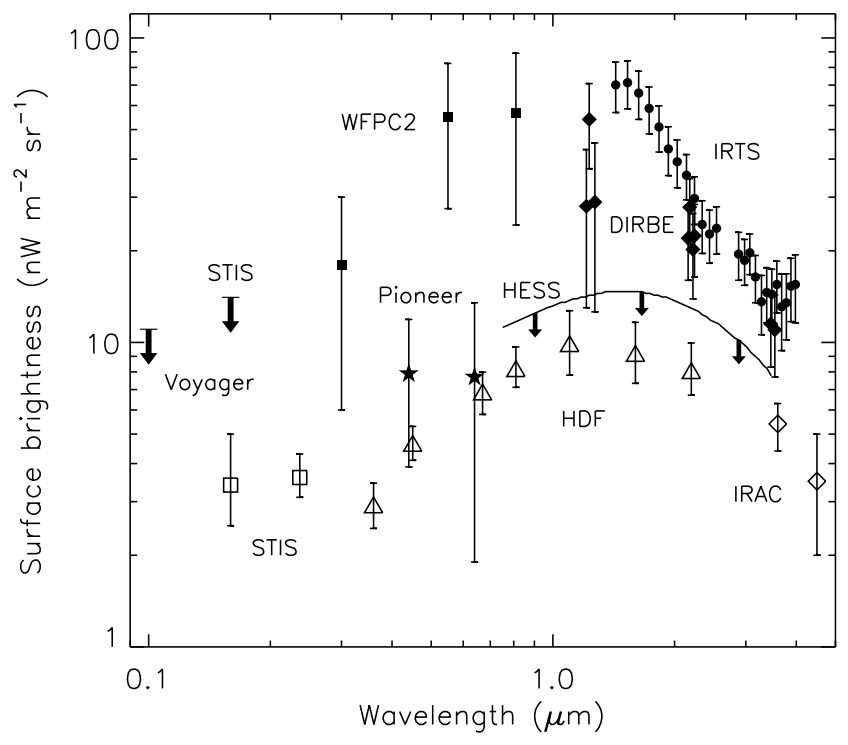

Fig. 2. Current measurements of the cosmic background (filled symbols) and the integrated brightness of galaxies (open symbols). At optical wavelengths, squares and stars represent the results by Bernstein (2007) and Matsuoka et al. (2011a), respectively. The solid line with arrows between 0.8 and $4 \mu \mathrm{m}$ represent the upper limits reported by Aharonian et al. (2006). The integrated brightness of galaxies at the corresponding wavelengths are given by Madau \& Pozzetti (2000) (triangles). Further details of this figure are found in Matsuoka et al. (2011a).

of the upper limits found by Aharonian et al. (2006). With our best estimates, approximately $60 \%$ and $90 \%$ of the COB have already been resolved into discrete galaxies in the HDF at $0.44 \mu \mathrm{m}\left(B_{\text {IPP }}\right)$ and $0.64 \mu \mathrm{m}$ $\left(R_{\text {IPP }}\right)$, respectively. On the other hand, Totani et al. (2001) demonstrated that $60-90 \%$ and $80-100 \%$ of the total light from galaxies have been resolved at 0.45 and $0.61 \mu \mathrm{m}$. The above facts indicate that the bulk of the COB is comprised of normal galaxies, and there is little room for contributions of other populations, such as "first stars", at these wavelengths.

\section{ACKNOWLEDGEMENTS}

This work was supported by Grants-in-Aid for Young Scientists (21840027, 22684005), Specially Promoted Research on Innovative Areas (22111503), and the Global COE Program of Nagoya University "Quest for Fundamental Principles in the Universe" from JSPS and MEXT of Japan. 


\section{REFERENCES}

Aharonian, F., Akhperjanian, A. G., Bazer-Bachi, A. R., et al., 2006, A Low Level of Extragalactic Background Light as Revealed by $\gamma$-rays from Blazars, Nature, 440, 1018

Aumann, H. H., Fowler, J. W., \& Melnyk, M., 1990, A Maximum Correlation Method for Image Construction of IRAS Survey Data, AJ, 99, 1674

Bernstein, R. A., 2007, The Optical Extragalactic Background Light: Revisions and Further Comments, ApJ, 666, 663

Bernstein, R. A., Freedman, W. L., \& Madore, B. F., 2002, The First Detections of the Extragalactic Background Light at 3000, 5500, and $8000 \AA$ A. I. Results, ApJ, 571, 56

Girardi, L., Groenewegen, M. A. T., Hatziminaoglou, E., \& da Costa, L., 2005, Star Counts in the Galaxy. Simulating from Very Deep to Very Shallow Photometric Surveys with the TRILEGAL Code, A\&A, 436, 895

Gordon, K. D., Witt, A. N., \& Friedmann, B. C., 1998, Detection of Extended Red Emission in the Diffuse Interstellar Medium, ApJ, 498, 522

Hanner, M. S., Weinberg, J. L., Deshields, L. M., II, Green, B. A., \& Toller, G. N., 1974, Zodiacal Light and the Asteroid Belt: The View from Pioneer 10, J. Geophys. Res., 79, 3671

Høg, E., Fabricius, C., Makarov, V. V., et al., 2000, The Tycho-2 Catalogue of the 2.5 Million Brightest Stars, A\&A, 355, L27

Lagache, G., Haffner, L. M., Reynolds, R. J., \& Tufte, S. L., 2000, Evidence for Dust Emission in the Warm Ionised Medium Sing WHAM Data, A\&A, 354,247

Lasker, B. M., Lattanzi, M. G., McLean, B. J., et al., 2008, The Second-Generation Guide Star Catalog: Description and Properties, AJ, 136, 735

Leinert, C., Bowyer, S., Haikala, L. K., et al., 1998, The 1997 Reference of Diffuse Night Sky Brightness, A\&AS, 127, 1

Madau, P. \& Pozzetti, L., 2000, Deep Galaxy Counts, Extragalactic Background Light and the Stellar Baryon Budget, MNRAS, 312, L9

Matsuoka, Y., 2012, Co-evolution of Galaxies and Central Black Holes: Observational Evidence on the Trigger of AGN Feedback, ApJ, 750, 54

Matsuoka, Y. \& Kawara, K., 2010, Witnessing the Active Assembly Phase of Massive Galaxies Since $\mathrm{z}=1$, MNRAS, 405, 100
Matsuoka, Y., Kawara, K., \& Oyabu, S., 2008, LowIonization Emission Regions in Quasars: Gas Properties Probed with Broad O I and Ca II Lines, ApJ, 673, 62

Matsuoka, Y., Ienaka, N., Kawara, K., \& Oyabu, S., 2011a, Cosmic Optical Background: The View from Pioneer 10/11, ApJ, 736, 119

Matsuoka, Y., Masaki, S., Kawara, K., \& Sugiyama, N., 2011b, Halo Occupation Distribution of Massive Galaxies Since z $=1$, MNRAS, 410, 548

Matsuoka, Y., Oyabu, S., Tsuzuki, Y., \& Kawara, K., 2007, Observations of O I and Ca II Emission Lines in Quasars: Implications for the Site of $\mathrm{Fe}$ II Line Emission, ApJ, 663, 781

Schlegel, D. J., Finkbeiner, D. P., \& Davis, M., 1998, Maps of Dust Infrared Emission for Use in Estimation of Reddening and Cosmic Microwave Background Radiation Foregrounds, ApJ, 500, 525

Totani, T., Yoshii, Y., Iwamuro, F., Maihara, T., \& Motohara, K., 2001, Diffuse Extragalactic Background Light Versus Deep Galaxy Counts in the Subaru Deep Field: Missing Light in the Universe?, ApJ, 550, L137

Weinberg, J. L., Hanner, M. S., Beeson, D. E., Deshields, L. M., II, \& Green, B. A., 1974, Background Starlight Observed from Pioneer 10, J. Geophys. Res., 79, 3665 